\title{
The clinical significance of UBE2C gene in progression of renal cell carcinoma
}

\author{
Zhiping Chen, ${ }^{1}$ Lanfeng Wang ${ }^{2}$ \\ ${ }^{1}$ Department of Laboratory Medicine; \\ ${ }^{2}$ Department of Nephrology, First affiliated Hospital of Gannan Medical University, Ganzhou, China
}

\begin{abstract}
Renal cell carcinoma (RCC), with high morbidity and mortality, is one of the top ten serious cancers. Due to limited therapies and little knowledge about the mechanism underlying RCC, overall survival of RCC patients is poor. UBE2C is a member of ubiquitin modification system and promotes carcinogenesis in cancer, but its role in RCC is unknown. Based on the TCGA (The Cancer Genome Atlas) data, UBE2C was over-expressed in a total of $525 \mathrm{RCC}$ tissues and displayed higher expression in advanced tissues (stage IV $v s$ stage I, $\mathrm{p}<0.05$ ). RT-qPCR and IHC analysis confirmed over-expression of UBE2C in 90 of clinical RCC tissues. Further, UBE2C was associated with clinical factors including TNM stage, gender, and pathological stage. And higher UBE2C expression predicted shorter overall survival and progression-free survival. Both univariate and multivariate COX analysis suggested $U B E 2 C$ as a critical gene in RCC. Then GO and KEGG analysis showed that cell cycle and DNA replication pathways were two top signaling pathways affected by UBE2C. In vitro assay showed that knockdown of $U B E 2 C$ in $786-O$ cells inhibited proliferation and migration significantly. Therefore, this study proves that $U B E 2 C$ is an important gene in RCC and is essential to proliferation and migration of RCC.
\end{abstract}

Key words: $U B E 2 C$; GO analysis; KEGG analysis; renal cell carcinoma.

Correspondence: Dr. Lanfeng Wang, MD, Department of Nephrology, First affiliated Hospital of Gannan Medical University, Jiangxi, China. E-mail: lanfengwang@gmx.com

Contributions: LW; study design, draft review; ZC, data acquisition and analysis, manuscript drafting. Both authors read and approved the final version of the manuscript and agreed to be accountable for all aspects of the work.

Funding: This research was supported by Natural Science Foundation of Jiangxi Province (No.20192BAB205075 and No.20202BABL206119) and Health Commission of Jiangxi Province (No.20204518).

Conflict of interest: The authors declare that they have no competing interests, and all authors confirm accuracy.

Availability of data and materials: The data used to support the findings of this study are available from the corresponding author upon request.

Ethical Approval: Not applicable. 


\section{Introduction}

Renal cell carcinoma (RCC) is a malignant disease and originates from renal parenchyma urinary tubule epithelial system. ${ }^{1}$ Among them, clear cell carcinomas the major type and accounts for about $70 \%$ of all RCC patients. ${ }^{1-3}$ The main therapy for RCC is resection assisted with chemo-radiotherapy. But RCC is not so sensitive to radiotherapy or chemotherapytherapy. ${ }^{3}$ In recent years, target therapy is used to treat RCC and about $20-40 \%$ of RCC patients could benefit from target therapy. ${ }^{4,5}$ However, long-term survival is not easily acquired. ${ }^{6}$ According to the latest report released by AACR in 2019 , the five-year survival rate for patients with distant metastasis is only $12 \% .^{7}$ And about $16 \%$ of patients are diagnosed with distant metastasis. In addition, a total of 73,820 persons will be subjected to kidney cancer and about 14,000 will die. ${ }^{7}$ This is partially due to the ignorance of mechanism underlying $\mathrm{RCC}$.

Ubiquitin-conjugating enzyme $\mathrm{E} 2 \mathrm{C}$ (UBE2C) is a member of the ubiquitin coupling enzyme E2 family and interacts with specific E3 enzymes, which leads to degradation of substrate proteins. ${ }^{8}$ The substrate proteins of UBE2C are cell cycle-related proteins involved in regulation of cell mitosis. Recent studies indicated that $U B E 2 C$ played an oncogenic role in several types of cancer. For example, the abnormal expression of UBE2C caused chromosomal instability and participated in development and progression in gastric cancer. ${ }^{9}$ $U B E 2 C$ is expressed abundantly in breast cancer, ${ }^{10}$ colon cancer, ${ }^{11}$ ovarian cancer, ${ }^{12}$ and liver cancer. ${ }^{11}$ And $U B E 2 C$ was shown to be closely correlated with tumor stage, which suggests that UBE2C might be a new tumor marker and a promising therapeutic target in these tumors. ${ }^{10-13}$ However, the expression of UBE2C in RCC and its clinical value remain unclear. The cancer genome atlas (TCGA) database is a public database consisted of cancer genomics from over 20,000 cases of primary cancer and matched normal samples spanning 33 cancer types. ${ }^{14}$ The database contributes greatly to the research on elucidation of molecular mechanism underlying tumorigenesis. ${ }^{15}$

In order to evaluate the clinical significance of $U B E 2 C$ in $\mathrm{RCC}$, we extracted the clinical information of RCC patients and UBE2C expression profile in RCC tissues from TCGA database. Then the relationship of UBE2C with pathological factors and prognoses of RCC patients was analyzed. And the potential process as well as signaling pathways were explored.

\section{Materials and Methods}

\section{Bioinformatics analysis and TCGA mining}

In order to analyze the differential expression levels of $U B E 2 C$ in RCC and normal renal tissues, TCGA database was extracted. The mRNA expression level of UBE2C from a total of 525 cases of RCC and 72 cases of normal renal tissues was downloaded from the TCGA database (https://cancergenome.nih.gov/). Then, RStudio (Version 1.1.442), a free and open-source data analysis software, was used to analyze the differential expression of $U B E 2 C$ and to deduce the overall survival (OS) and disease-free survival (DFS) curves. Gene ontology (GO) and Kyoto Encyclopedia of Genes and Genomes (KEGG) were used in conjunction with DAVID 6.8 and Enrichment Map plug-in Cytoscape to visualize the significant pathways and differential expression genes (DEGs) in RCC.

\section{RCC tissues and pathological information of patients}

A total of 90 RCC tissues and matched normal tissues were retrospectively collected to perform RT-qPCR analysis. All the patients had received radical operation in our hospital from July 2006 to December
2010.The last follow-up time was August 2017. The pathologic diagnosis of all cases was renal clear cell carcinoma after operation. This study was approved by the Ethics Committee of First affiliated Hospital of Gannan Medical University and written consent was collected from all patients.

\section{RT-qPCR}

Total RNA of renal cancer tissues and matched normal tissues was extracted with TRIzol (Invitrogen, USA) and treated with RNase-free DNase (Promega, USA). SYBR Green quantitative real-time PCR was performed by Stepone Real-time PCR system (Applied Biosystems 7000 , USA). Beta-actin was used as the internal control. Relative expression level of each gene was calculated by the $2^{-\Delta \Delta \mathrm{Ct}}$ method, in which $\Delta \Delta \mathrm{Ct}=$ Test group $\Delta \mathrm{Ct}\left(\mathrm{CT}_{\text {interested gene }}-\mathrm{CT}_{\text {Beta-actin }}\right)$ - Control group $\Delta \mathrm{Ct}\left(\mathrm{CT}_{\text {interested gene }}-\mathrm{CT}_{\text {Beta-actin }}\right)$.

\section{Immunohistochemical analysis}

Human tumor tissues were fixed in $4 \%$ paraformaldehyde, embedded in paraffin and sectioned as $5 \mu \mathrm{m}$. Each sectioned tissues were incubated with antibody against UBE2C (1:500, ab252940, Abcam, NY, USA) diluted with $0.01 \mathrm{M}$ PBST buffer (0.01 M PBS, $0.001 \times$ Triton $\mathrm{X}-100)$ for $12 \mathrm{~h}$ at $44^{\circ} \mathrm{C}$ followed by HRP-conjugated secondary antibody (1:100, Beyotime, Shanghai, China). Peroxidase conjugates were determined using 3,3'-diaminobenzidine solution.

\section{RNA interference assay (RNAi)}

siRNA fragments targeting human UBE2C gene (siUBE2C: 5'TCCTTTTTGTGATTTCTGTATAG-3') was designed and synthesized. Random sequence was designed as negative control (NC). Then RCC typical cells 786-O cells were cultured in a 6-well plate and transduced with siUBE2C or NC (General Biosystems, Anhui, China) by Lipo reagent (Yeasen, Shanghai, China) for 4-6 $\mathrm{h}$ according to the manufacture's instruction. The knockdown efficiency of siUBE2C was tested by RT-qPCR method.

\section{Cell proliferation assay}

Treated cells were seeded into a 96 -well plate at $5 \times 10^{3}$ cell $/$ well and cultured consecutively for $96 \mathrm{~h}$. At each designed time point, $10 \mu \mathrm{L}$ of CCK-8 agent was added and cultured for another 1-2 $\mathrm{h}$. Then OD value at $450 \mathrm{~nm}$ was detected on microplate reader (Molecular Devices, CA, USA). The proliferation rate of cells was calculated according to the negative control.

\section{Cell invasion assay}

Transwell rooms with 8 - $\mu$ m-pore size membrane were pre-treated with $0.2 \mathrm{~mL}$ Matrigel $12 \mathrm{~h}$ before seeding tumor cells. Then a total of $2 \times 10^{4}$ cells/well was seeded into upper room and DMEM medium without FBS was added. DMEM medium containing15\% FBS was added in the lower room. After culture for $24 \mathrm{~h}$, cells on the upper room were removed and cells in the lower surface of upper room were fixed by $4 \%$ paraformaldehyde for $30 \mathrm{~min}$ and dyed with $0.5 \%$ crystal violet for 10 min. Then stained cells were observed under a microscope and counted.

\section{Statistical analysis}

SPSS 16.0software (SPSS, Inc., Chicago, IL, USA) was used to analyze the experimental data. All data was expressed as mean $\pm \mathrm{SD}$. TCGA data were analyzed by the independent samples $t$-test to compare the differential levels of UBE2C mRNA between RCC and control. Chi-square test or Fisher's exact test was employed to analyze the relationship between $U B E 2 C$ expression and clinicopathological factors of RCC patients. Kaplan-Meier analysis was used to produce the survival curve. Univariate and Cox multivariate survival analyses were performed to analyze the prognostic significance of $U B E 2 C$ in RCC patients. The difference was considered statistically significant when the $\mathrm{p}$-value was less than 0.05 . 


\section{Results}

\section{$U B E 2 C$ was highly expressed in RCC}

To explore the role of UBE2C in RCC, a total of $525 \mathrm{RCC}$ cases and 72 normal cases from TCGA database (https://cancergenome.nih.gov/) were extracted for further analysis. The clinical information of the $525 \mathrm{RCC}$ cases was displayed in Table 1. In brief, 187 cases were diagnosed as T3/T4 stage, accounted for $35.62 \%$ of total patients. About 205 cases were in stage III/IV, accounted for $39.05 \%$. And 157 cases were dead, accounted for $29.9 \%$. The mRNA level of UBE2C was significantly higher in RCC tissues than the control $(\mathrm{p}<0.05)$ (Figure 1A). The mean level of $U B E 2 C$ in RCC was 5 folds of that in normal control. Furthermore, the expression level of UBE2C in stage IV patients was remarkably higher than that in stage I patients (Figure 1B).

To verify the bioinformatics data, the mRNA expression of $U B E 2 C$ in 90 of RCC tissues and matched control was determined by RT-qPCR method. In consistency, UBE2C was expressed much higher in tumor tissues than the control and immunohistochemical (IHC) analysis confirmed this result (Figure 1C and 1D). Therefore, UBE2C was over-expressed in RCC tissues.

A

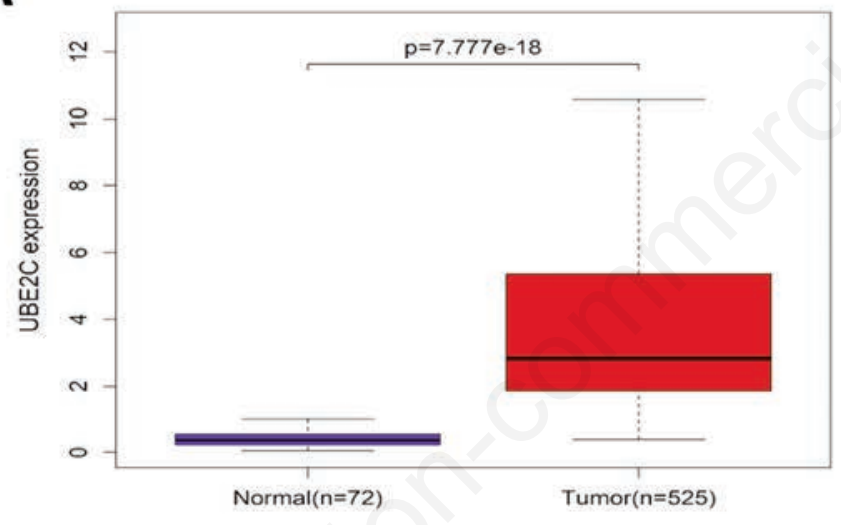

B

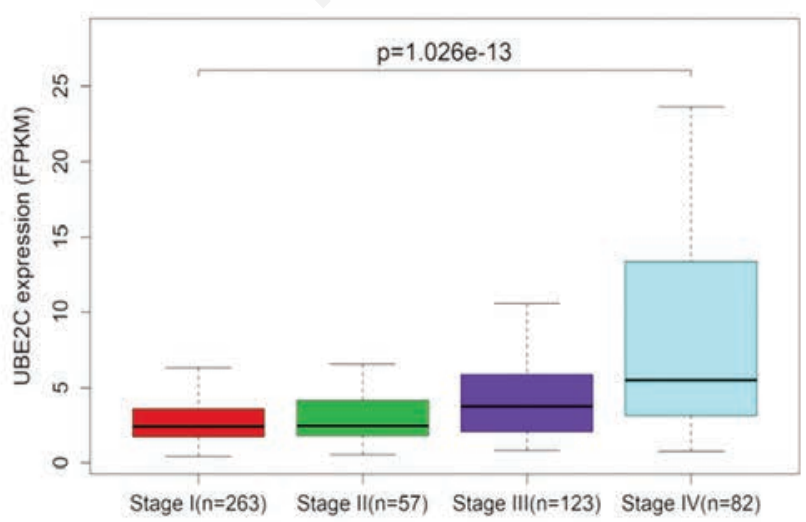

Table 1. Clinical parameters of RCC patients from TCGA database.

\begin{tabular}{lcc} 
Covariates & Type & Stat \\
T & T1 & $269(51.24 \%)$ \\
& T2 & $69(13.14 \%)$ \\
& T3 & $176(33.52 \%)$ \\
N & T4 & $11(2.1 \%)$ \\
& N0 & $238(45.33 \%)$ \\
& N1 & $15(2.86 \%)$ \\
M & NX & $272(51.81 \%)$ \\
& M0 & $419(79.81 \%)$ \\
Stage & M1 & $77(14.67 \%)$ \\
& MX & $29(5.52 \%)$ \\
& Stage I & $263(50.1 \%)$ \\
& Stage II & $57(10.86 \%)$ \\
Fustat & Stage III & $123(23.43 \%)$ \\
& Stage IV & $82(15.62 \%)$ \\
Age & Alive (0) & $368(70.1 \%)$ \\
& Dead (1) & $157(29.9 \%)$ \\
\hline
\end{tabular}

T1-4, primary tumor; N0, no regional lymph node involved; N1, few regional lymph nodes involved NX, regional lymph node unevaluated; M0, no distant metastasis; M1, distant metastasis; MX, distant metastasis unevaluated; the percentage in parentheses means the number in the total cases; fustat is a parameter in survival analysis: fustat 1 indicated alive, fustat 0 indicated dead.
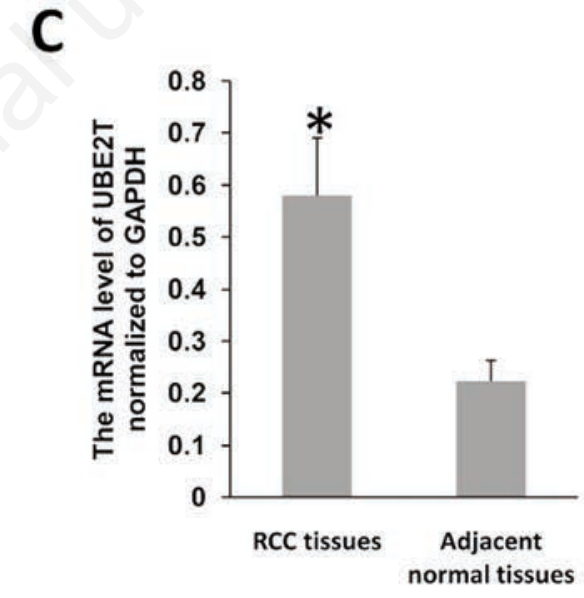

D

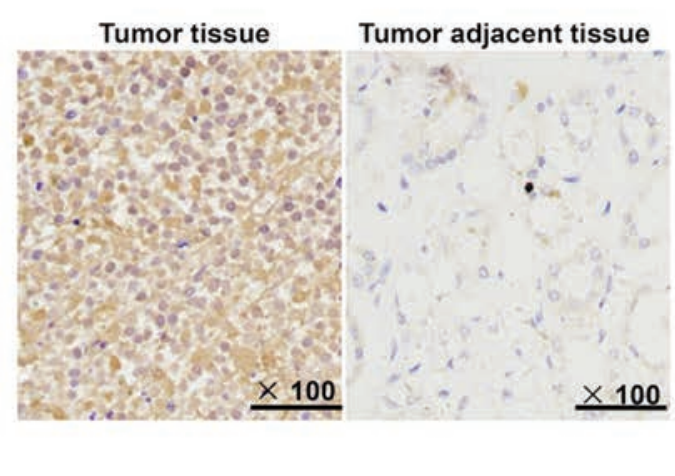

Figure 1. UBE2C was over-expressed in RCC. A) Expression profiles of UBE2C extracted from TCGA database. B) The expression of UBE2C in RCC patients with different pathological stage. C) The mRNA level of UBE2C in a total of 90 RCC tissues and adjacent normal control by RT-qPCR assay. D) The IHC analysis of UBE2C expression in RCC tissues and matched control; ${ }^{*} \mathrm{p}<0.05$ was considered as statistically significant. 


\section{$U B E 2 C$ was correlated to clinicopathological factors in RCC patients}

Tumor is a malignant disease caused by multiple factors and genetic gene is a key factor. Driver gene is a critical genetic factor in tumor. In this study, RCC patients were separated as low and high group based on the expression of $U B E 2 C$. The cut value is 2fold ( $U B E 2 C$ expression in RCC tissues to control tissues). Then chi-square test was performed to analyze the relationship of UBE2C level with clinical factors in RCC patients. As shown in Table 2, UBE2C expression was associated with TNM stage, pathological stage, gender and survival but not age in RCC patients. ${ }^{16}$ Briefly, UBE2C was expressed at high level in $45.42 \%$ of T3 patients while at low level in $21.67 \%$ of T3 patients. But it was $38.93 \%$ vs $63.5 \%$ in $\mathrm{T} 1$ patients.

In stage I, $37.79 \%$ patients displayed high $U B E 2 C$ expression when it was $62.36 \%$ in low UBE2C expression. But it was $29.01 \%$ vs $17.87 \%$ in stage III and $24.43 \%$ vs $6.84 \%$ in stage IV patients. Moreover, death rate of patients with high $U B E 2 C$ expression is $41.98 \%$ but it was $17.87 \%$ in low UBE2C RCC patients. In 90 clin- ical cases, Clinicopathological analysis demonstrated that $U B E 2 C$ was correlated to TNM stage and pathological stage in RCC (Table $3)$. Conclusively, $U B E 2 C$ displayed important clinic role in progression of RCC.

\section{$U B E 2 C$ showed prognostic value in $\mathrm{RCC}$}

OS and progression-free survival (PFS) are two critical indicators in cancer. By mining TCGA database, we found that UBE2C expression was associated with OS and PFS in RCC patients. As shown in Figure 2A, RCC patients with higher $U B E 2 C$ expression $(\mathrm{n}=262)$ showed much shorter OS than those with lower UBE2C expression $(\mathrm{n}=263)(\mathrm{p}<0.05)$. And patients with lower $U B E 2 C$ expression $(n=262)$ displayed much better PFS than the control (Figure 2B, $n=263)(p<0.05)$. By multivariate COX analysis, we found that patients with higher UBE2C expression exhibited worse OS than the control $(\mathrm{p}<0.001, \mathrm{HR}=1.01,95 \%$ CI $1.006-1.020)$ (Tables 3 and 4). In addition, pathological stage was associated with patient survival. Therefore, $U B E 2 C$ might be a critical factor for predicting the prognosis of RCC patients.

Table 2. Correlation analysis of UBE2C expression with clinical parameters of RCC patients based on TCGA data.

\begin{tabular}{|c|c|c|c|c|c|}
\hline Covariates & Type & Count & Low & High & p \\
\hline Fustat & $\begin{array}{l}\text { Alive (0) } \\
\text { Dead (1) }\end{array}$ & $\begin{array}{l}368(70.1 \%) \\
157(29.9 \%)\end{array}$ & $\begin{array}{c}216(82.13 \%) \\
47(17.87 \%)\end{array}$ & $\begin{array}{l}152(58.02 \%) \\
110(41.98 \%)\end{array}$ & 2.87E-09 \\
\hline Gender & $\begin{array}{l}\text { Female } \\
\text { Male }\end{array}$ & $\begin{array}{l}184(35.05 \%) \\
341(64.95 \%)\end{array}$ & $\begin{array}{l}107(40.68 \%) \\
156(59.32 \%)\end{array}$ & $\begin{array}{c}77(29.39 \%) \\
185(70.61 \%)\end{array}$ & 0.008775808 \\
\hline $\mathrm{T}$ & $\begin{array}{l}\text { T1 } \\
\text { T2 } \\
\text { T3 } \\
\text { T4 }\end{array}$ & $\begin{array}{c}269(51.24 \%) \\
69(13.14 \%) \\
176(33.52 \%) \\
11(2.1 \%)\end{array}$ & $\begin{array}{c}167(63.5 \%) \\
38(14.45 \%) \\
57(21.67 \%) \\
1(0.38 \%)\end{array}$ & $\begin{array}{c}102(38.93 \%) \\
31(11.83 \%) \\
119(45.42 \%) \\
10(3.82 \%)\end{array}$ & $6.83 \mathrm{E}-10$ \\
\hline $\mathrm{N}$ & $\begin{array}{l}\text { N0 } \\
\text { N1 } \\
\text { N2 }\end{array}$ & $\begin{array}{c}238(45.33 \%) \\
15(2.86 \%) \\
272(51.81 \%)\end{array}$ & $\begin{array}{c}116(44.11 \%) \\
2(0.76 \%) \\
145(55.13 \%)\end{array}$ & $\begin{array}{c}122(46.56 \%) \\
13(4.96 \%) \\
127(48.47 \%)\end{array}$ & 0.009062443 \\
\hline M & $\begin{array}{l}\text { M0 } \\
\text { M1 } \\
\text { M2 }\end{array}$ & $\begin{array}{c}419(79.81 \%) \\
77(14.67 \%) \\
29(5.52 \%)\end{array}$ & $\begin{array}{c}226(85.93 \%) \\
16(6.08 \%) \\
21(7.98 \%)\end{array}$ & $\begin{array}{c}193(73.66 \%) \\
61(23.28 \%) \\
8(3.05 \%)\end{array}$ & $2.88 \mathrm{E}-08$ \\
\hline Stage & $\begin{array}{l}\text { Stage I } \\
\text { Stage II } \\
\text { Stage III } \\
\text { Stage IV }\end{array}$ & $\begin{array}{c}263(50.1 \%) \\
57(10.86 \%) \\
123(23.43 \%) \\
82(15.62 \%)\end{array}$ & $\begin{array}{c}164(62.36 \%) \\
34(12.93 \%) \\
47(17.87 \%) \\
18(6.84 \%)\end{array}$ & $\begin{array}{c}99(37.79 \%) \\
23(8.78 \%) \\
76(29.01 \%) \\
64(24.43 \%)\end{array}$ & $5.32 \mathrm{E}-11$ \\
\hline Age & $\begin{array}{l}<=60 \\
<60\end{array}$ & $\begin{array}{l}260(49.52 \%) \\
265(50.48 \%)\end{array}$ & $\begin{array}{l}132(50.19 \%) \\
131(49.81 \%)\end{array}$ & $\begin{array}{l}128(48.85 \%) \\
134(51.15 \%)\end{array}$ & 0.826927487 \\
\hline
\end{tabular}

The percentage in the parentheses means the number in the total cases or in each group; p-value less than 0.05 displays significant correlation of UBE2C expression with clinical factor.

Table 3. Univariate COX analysis of 525 RCC patients from TCGA database.

\begin{tabular}{lcccc} 
Term & HR & HR (lower 0.95) & HR (upper 0.95) & p-value \\
Gender & 0.945597502 & 0.683103292 & 1.308959635 & 0.735990786 \\
$\mathrm{~T}$ & 2.051550134 & 1.723523146 & 2.442008372 & $6.27 \mathrm{E}-16$ \\
\hline $\mathrm{N}$ & 0.867947038 & 0.739824998 & 1.018257105 & 0.082220867 \\
$\mathrm{M}$ & 2.483956418 & 1.930133225 & 3.196690987 & $1.56 \mathrm{E}-12$ \\
\hline Stage & 1.956212786 & 1.702382614 & 2.247889771 & $3.00 \mathrm{E}-21$ \\
Age & 1.028451721 & 1.015156215 & 1.041921359 & $2.38 \mathrm{E}-05$ \\
\hline UBE2C & 1.018830727 & 1.012673308 & 1.025025585 & $1.62 \mathrm{E}-09$ \\
\hline
\end{tabular}

HR, hazard ratio. 
Identification of potential signaling pathways associated with $U B E 2 C$ in RCC

Based on the differential expressed genes (DEGs) extracted from TCGA database, we explored the signaling pathways involved in development of RCC by GO and KEGG analysis. As seen in Figure $3 \mathrm{~A}$ and Table 5, GO analysis revealed that DEGs were mostly distributed in cell division $(n=86)$, mitotic nuclear division $(n=67)$, and sister chromatid cohesion $(\mathrm{n}=45)$ process. KEGG analysis revealed significance of $U B E 2 C$ in about 17 signal pathways in which cell cycle was the most important pathway $(\mathrm{p}<0.001)$ (Figure 3B). About 40 DEGs were enriched in cell cycle (Table 6). Then a total of 13 DEGs was enriched in DNA replication and p53 signaling pathway (Figure 3B, Table 6). It is known to us that the signaling pathways including cell cycle, cell division, p53 signaling all play important roles in tumor. So it is conceived that $U B E 2 C$ might regulate one or several of these pathways in RCC.

Table 4. Multivariate COX analysis of 525 RCC patients from TCGA database.

\begin{tabular}{lcccc} 
Term & HR & HR (lower 0.95) & HR (upper 0.95) & p-value \\
Gender & 1.052653432 & 0.748571991 & 1.480257425 & 0.7679768 \\
$\mathrm{~T}$ & 0.861615988 & 0.587843984 & 1.262889697 & 0.445157108 \\
\hline $\mathrm{N}$ & 0.893264318 & 0.758286997 & 1.052268001 & 0.176881432 \\
$\mathrm{M}$ & 1.08647467 & 0.669831613 & 1.762274555 & 0.736802667 \\
\hline Stage & 2.081291149 & 1.435882277 & 3.016802225 & 0.000108772 \\
Age & 1.033098721 & 1.017936353 & 1.048486935 & $1.58 \mathrm{E}-05$ \\
\hline UBE2C & 1.013312908 & 1.006346286 & 1.020327757 & 0.00017179 \\
\hline
\end{tabular}

HR, hazard ratio.

Table 5. GO enrichment analysis of differential expression genes in RCC.

\begin{tabular}{llccc}
\hline Id & Term & Count & p-value & padjust \\
G0:0051301 & Cell division & 86 & $6.50 \mathrm{E}-48$ & $1.13 \mathrm{E}-44$ \\
G0:0007067 & Mitotic nuclear division & 67 & $6.75 \mathrm{E}-40$ & $1.17 \mathrm{E}-36$ \\
\hline G0:0007062 & Sister chromatid cohesion & 45 & $5.50 \mathrm{E}-37$ & $9.54 \mathrm{E}-34$ \\
G0:0005654 & Nucleoplasm & 212 & $3.13 \mathrm{E}-33$ & $4.47 \mathrm{E}-30$ \\
\hline GO:0006260 & DNA replication & 47 & $2.50 \mathrm{E}-30$ & $4.34 \mathrm{E}-27$ \\
GO:0000777 & Condensed chromosome kinetochore & 31 & $9.70 \mathrm{E}-23$ & $1.38 \mathrm{E}-19$ \\
\hline G0:0000082 & G1/S transition of mitotic cell cycle & 33 & $2.79 \mathrm{E}-22$ & $4.84 \mathrm{E}-19$ \\
G0:0005515 & Protein binding & 415 & $5.73 \mathrm{E}-22$ & $8.67 \mathrm{E}-19$ \\
\hline G0:0005634 & Nucleus & 292 & $2.14 \mathrm{E}-21$ & $3.05 \mathrm{E}-18$ \\
G0:0000776 & Kinetochore & 29 & $2.41 \mathrm{E}-21$ & $3.45 \mathrm{E}-18$ \\
\hline
\end{tabular}

Count indicates the enriched genes in each bioprocess in Term.

Table 6. GO enrichment analysis of differential expression genes in RCC.

\begin{tabular}{llllc}
\hline ID & Description & Count & p-value & p adjust \\
hsa04110 & Cell cycle & 40 & $4.66 \mathrm{E}-28$ & $9.97 \mathrm{E}-26$ \\
hsa03030 & DNA replication & 13 & $1.48 \mathrm{E}-10$ & $1.59 \mathrm{E}-08$ \\
\hline hsa03460 & Fanconi anemia pathway & 13 & $3.96 \mathrm{E}-08$ & $2.83 \mathrm{E}-06$ \\
hsa04914 & Progesterone-mediated oocyte maturation & 17 & $7.11 \mathrm{E}-08$ & $3.81 \mathrm{E}-06$ \\
\hline hsa04114 & Oocyte meiosis & 18 & $4.11 \mathrm{E}-07$ & $1.51 \mathrm{E}-05$ \\
hsa05166 & Human T-cell leukemia virus 1 infection & 27 & $4.24 \mathrm{E}-07$ & $1.51 \mathrm{E}-05$ \\
\hline hsa04115 & p53 signaling pathway & 13 & $1.41 \mathrm{E}-06$ & $4.32 \mathrm{E}-05$ \\
hsa03410 & Base excision repair & 7 & 0.000142001 & 0.00379852 \\
\hline hsa04218 & Cellular senescence & 16 & 0.000204533 & 0.004863351 \\
hsa03013 & RNA transport & 16 & 0.000436647 & 0.009344253 \\
\hline hsa03440 & Homologous recombination & 7 & 0.000583551 & 0.011352725 \\
hsa05130 & Pathogenic Escherichia coli infection & 8 & 0.000730516 & 0.013027537 \\
\hline hsa03050 & Proteasome & 7 & 0.001041273 & 0.017140959 \\
hsa03430 & Mismatch repair & 5 & 0.001170701 & 0.017895004 \\
\hline hsa05203 & Viral carcinogenesis & 16 & 0.002452518 & 0.034989252 \\
hsa00240 & Pyrimidine metabolism & 10 & 0.003407413 & 0.045574151 \\
\hline
\end{tabular}




\section{$U B E 2 C$ is important for cell proliferation in $\mathrm{RCC}$}

To verify the real function of $U B E 2 C$ in RCC, $U B E 2 C$ was knocked down in 786-O cells. The knockdown efficiency of $U B E 2 C$ in $786-\mathrm{O}$ cells was over $66 \%$ (Figure $4 \mathrm{~A}$ ). Then by CCK-
8 assay, we found that the growth of 786-O cells was dramatically inhibited at third day of culture. The proliferation fold in $U B E 2 C$ knockdown group was only 1.53 but it was 3.49 in the control (Figure 4B). Therefore, UBE2C was essential to the growth of RCC cells.
A

\section{$\operatorname{UBE} 2 C(p=1.848 \mathrm{e}-07)$}

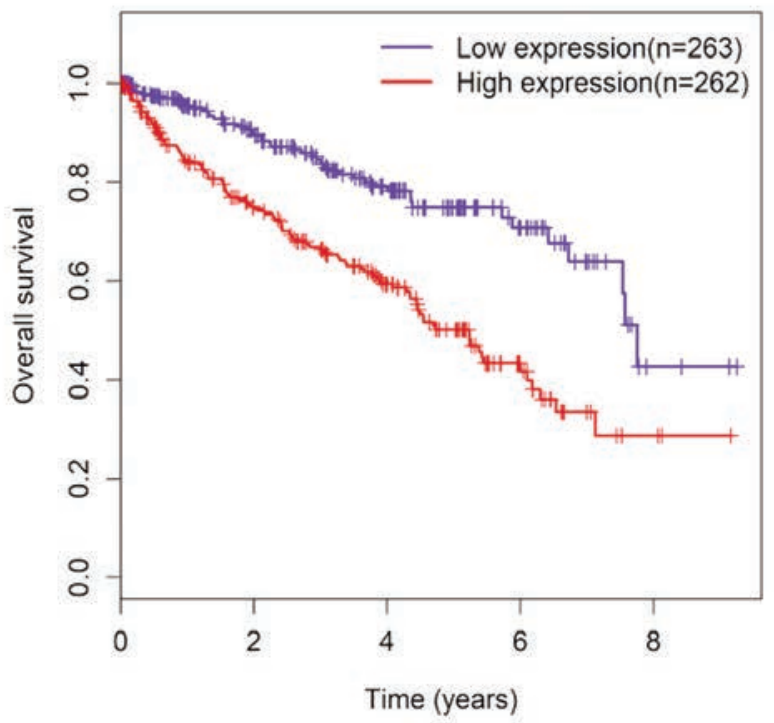

B

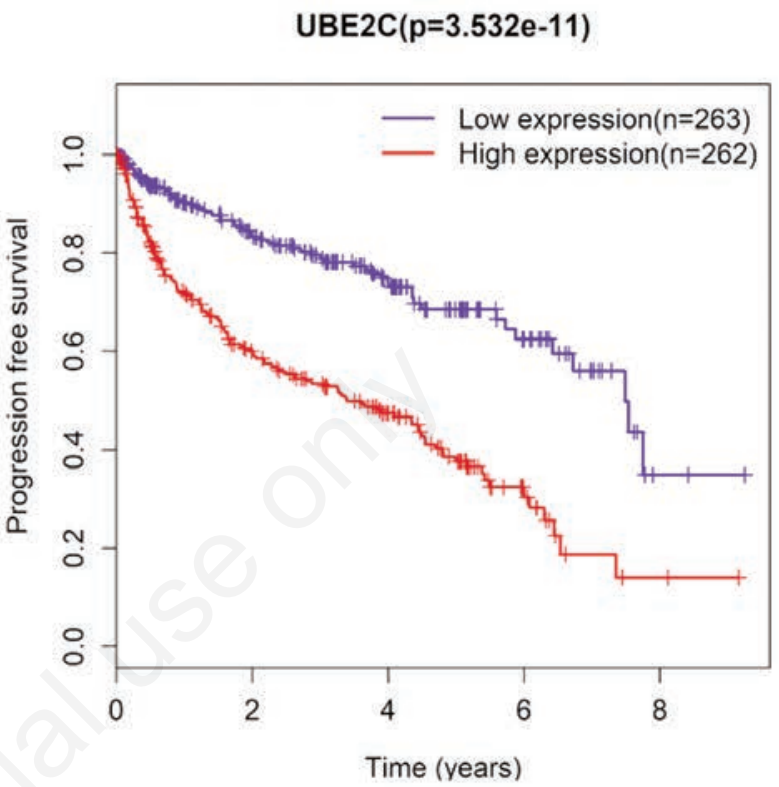

Figure 2. Overall survival (OS) and progression-free survival (PFS) curves of RCC patients based on the expression of UBE2C. A) OS curve based on TCGA data. B) PFS curve based on TCGA data.

A

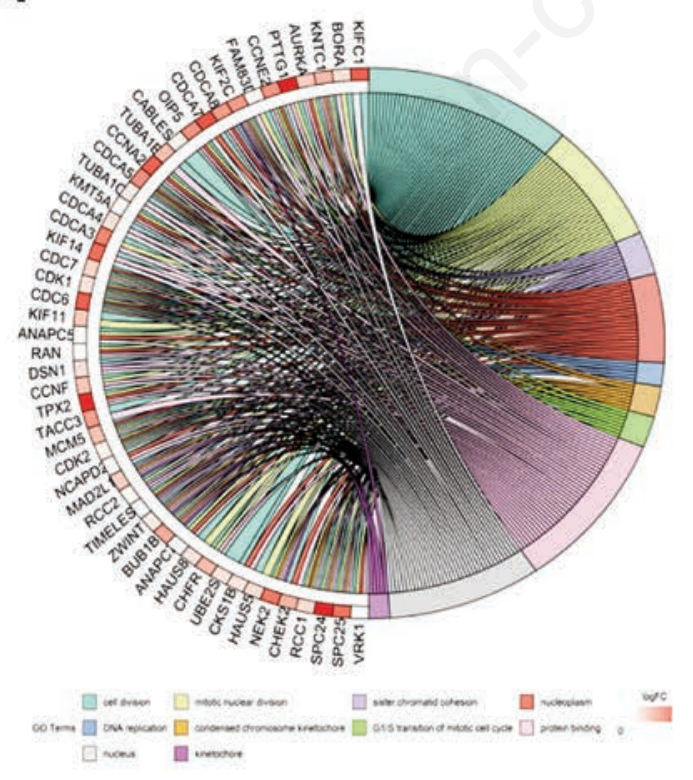

B

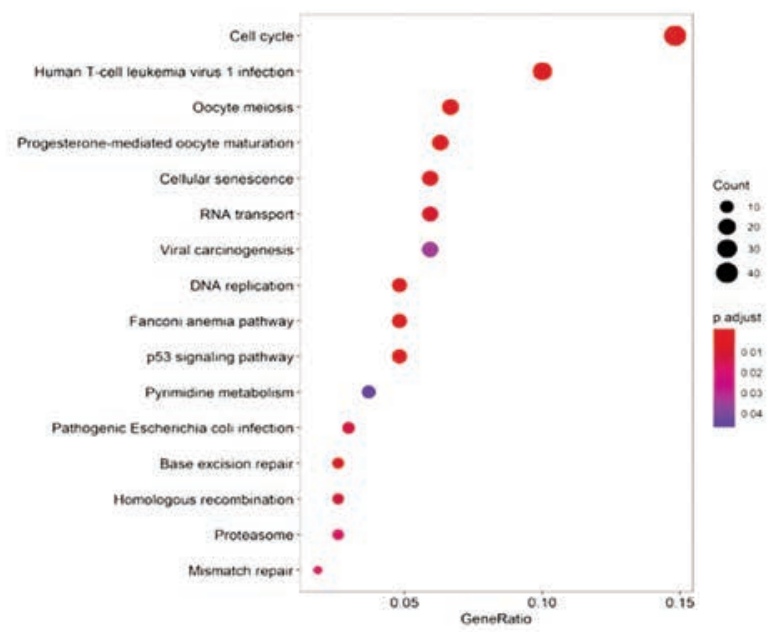

Figure 3. GO and KEGG analysis of UBE2C-associated pathways. A) GO analysis showed enrichment of differential expression genes. B) KEGG analysis showed the enriched signaling pathway. 


\section{$U B E 2 C$ contributes to cell migration in RCC}

Tumor cells often have potent migration ability according to the previous study. In transwell assay, we found that 786-O cells couldn't transmit through the chamber effectively. The number of transmitted cells in UBE2C knockdown group was decreased by $44 \%$ compared to the control (Figure 4 C,D). The results suggested that UBE2C conferred migration ability to $786-\mathrm{O}$ cells.

\section{Discussion}

$U B E 2 C$ is an important gene in cancer. For example, in prostate cancer, silence of UBE2C prevents cell growth. ${ }^{17-19}$ Methylation of $U B E 2 C$ lead to gene instability. ${ }^{18}$ Over-expression of $U B E 2 C$ is closely correlated to invasion and pathological stage in ovarian cancer. ${ }^{12}$ In this study, $U B E 2 C$ was firstly demonstrated to be clinically associated with progression and prognosis in RCC patients. This was consistent with previous studies in other cancers such as ovarian, gastric cancer. ${ }^{12,19}$

$U B E 2 C$ is also shown to be associated with poor prognosis in cancers. ${ }^{8,13,20,21}$ In this study, we demonstrated that patients with high $U B E 2 C$ expression displayed short survival time and decreased PFS, suggesting that $U B E 2 C$ might be a prognostic factor in RCC. This was further supported by multivariate COX analysis, which suggested $U B E 2 C$ as a critical gene in RCC. Taken together, $U B E 2 C$ played critical roles in development and progression of RCC and might be a new biomarker for diagnosis or prognosis in advanced RCC.

RCC is a malignant disease affected by multiple factors and a series of signaling pathways contributed to the initiation or progression of RCC. ${ }^{22,23}$ In this study, the DEGs between tumor tissues and normal tissues were extracted from the TCGA databases followed by GO enrichment analysis. As stated above, the most correlated process was cell division in which 86 DEGs were enriched. Then 67 DEGs were enriched in mitotic nuclear division and 47 DEGs in DNA replication process. The three processes are known to play important roles in embryonic development orhomeostasis. ${ }^{24,25}$ Aberrant in these processes often causes severe diseases or abnormalities such as cancer. So, it is possible that $U B E 2 C$ might also regulate cell division, DNA replication, and mitotic nuclear division in RCC. In KEGG analysis, 40 DEGs was enriched in cell cycle pathway while 13 DEGs in DNA replication pathway. Accelerated cell division was common in cancer and was associated with unlimited cell proliferation and accelerated cell cycle. DNA replication was also an active process in cancer. Here, KEGG analysis showed that cell cycle and DNA replication were two top pathways in RCC. Indeed, active DNA replication process provides basis for cell cycle transition. This will further contribute to the division and proliferation of cancer cells. As a result, we
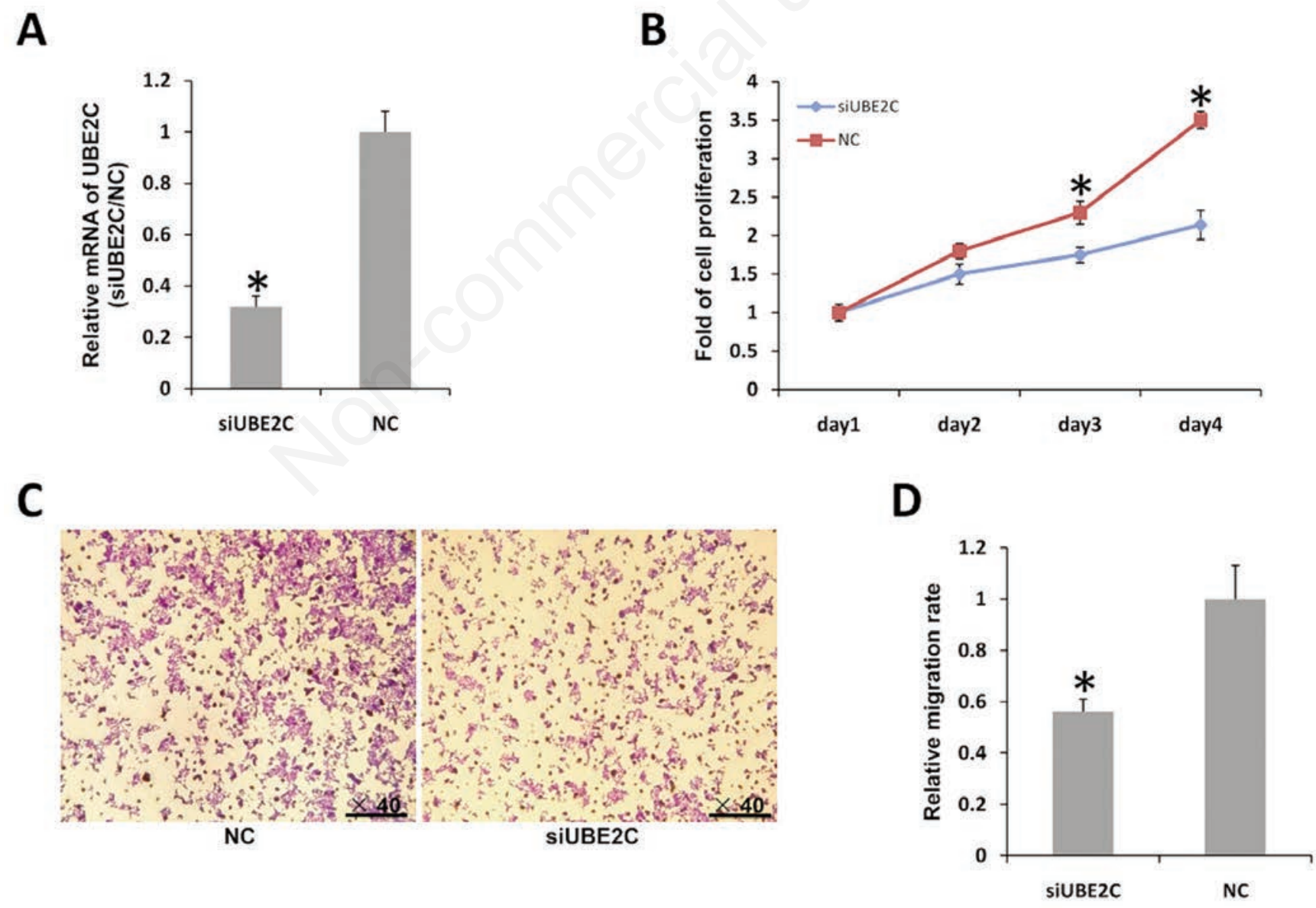

Figure 4. Knockdown of $U B E 2 C$ inhibited cell proliferation and migration in 786-O cells. A) Knockdown efficiency of $U B E 2 C$ in 786 $O$ cells determined by RT-qPCR technology. B) The proliferation curve of 786-O cells treated with siUBE2C or NC in CCK-8 assay for $96 \mathrm{~h}$. C,D) Transmitted cells stained by crystal violet in transwell assay; ${ }^{*} \mathbf{p}<0.05$ was considered as statistically significant. 
deduce that $U B E 2 C$ might regulate cell cycle and DNA replication pathway in RCC.

The bioinformatics analysis showed that $U B E 2 C$ played important roles in clinic in RCC. This was further supported by in vitro assay in 786-O cells. As stated in the above, knockdown of $U B E 2 C$ by RNNi significantly inhibited cell proliferation and migration in 786-O cells. It is known that potent proliferation and migration ability are two common traits in cancer. ${ }^{26,27}$ So, the preliminary data in 786-O cells further confirmed the role of $U B E 2 C$ in RCC. However, more experiments are needed to support the oncogenic role of $U B E 2 C$ in RCC and the mechanism that how $U B E 2 C$ promotes progression in RCC. In future, we plan to further detect the role of $U B E 2 C$ in more than two strain cell lines in RCC, especially the effects on cell apoptosis, cell cycle, and cell invasiveness. In addition, the in vivo role of $U B E 2 C$ in RCC is a very important proof to support this study.

In summary, this study proves the clinical role of $U B E 2 C$ and suggests it to be an important prognostic factor in RCC. UBE2C contributes to the proliferation and migration in RCC.

\section{References}

1. Nabi S, Kessler ER, Bernard B, Flaig TW, Lam ET. Renal cell carcinoma: a review of biology and pathophysiology. F1000Res 2018; 7:307.

2. Siegel RL, Miller KD, Jemal A. Cancer statistics, 2018. Ca Cancer J Clin 2018;68:7-30.

3. Vecchio SJD, Ellis RJ. Cabozantinib for the management of metastatic clear cell renal cell carcinoma. J Kidney Cancer VHL 2018;5:1-5.

4. Conti A, Santoni M, Amantini C, Burattini L, Berardi R, Santoni G, et al. Progress of molecular targeted therapies for advanced renal cell carcinoma. BioMed Res Int 2013;2013: 419176.

5. Flippot R, Escudier B, Albiges L. Immune checkpoint inhibitors: toward new paradigms in renal cell carcinoma. Drugs 2018;78:1443-57.

6. Sanchez-Gastaldo A, Kempf E, Aranzazu GDA, Duran I. Systemic treatment of renal cell cancer: A comprehensive review. Cancer Treat Rev 2017;60:77-89.

7. Siegel RL, Miller KD, Jemal A. Cancer Statistics, 2019. CA Cancer J Clin 2019;69:7-34

8. Hao Z, Zhang H, Cowell J. Ubiquitin-conjugating enzyme UBE2C: molecular biology, role in tumorigenesis, and potential as a biomarker. Tumour Biol 2012;33:723-30.

9. Zhang J, Liu XY, Yu GZ, Liu L, Wang JJ, Chen XY, et al. UBE2C is a potential biomarker of intestinal-type gastric cancer with chromosomal instability. Front Pharmacol 2018;9:847.

10. Han QL, Zhou C, Liu F, Xu GH, Zheng R, Zhang X. MicroRNA-196a post-transcriptionally upregulates the UBE2C proto-oncogene and promotes cell proliferation in breast cancer. Oncol Rep 2015;34:877-83.

11. Takahashi Y, Ishii Y, Nishida Y, Ikarashi M, Nagata T, Nakamura T, et al. Detection of aberrations of ubiquitin-conju- gating enzyme E2C gene (UBE2C) in advanced colon cancer with liver metastases by DNA microarray and two-color FISH. Cancer Genet Cytogen 2006;168:30-5.

12. Martinez-Canales S, Miguel LDR, Nuncia-Cantarero M,Paez R, Amir E, Gyorffy B, et al. Functional transcriptomic annotation and protein-protein interaction analysis identify EZH2 and UBE2C as key upregulated proteins in ovarian cancer. Cancer Med 2018;7:1896-907.

13. Loussouarn D, Campion L, Leclair F, Campone $M$, Charbonnel C, Ricolleau G, et al. Validation of UBE2C protein as a prognostic marker in node-positive breast cancer. $\mathrm{Br} \mathrm{J}$ Cancer 2009;101:166-73.

14. Wang Z, Jensen MA, Zenklusen JC. A Practical Guide to The Cancer Genome Atlas (TCGA). Methods Mol Biol 2016;1418:111-41

15. Cheng PF, Dummer R, Levesque MP. Data mining the cancer genome atlas in the era of precision cancer medicine. Swiss Med Wkly 2015;145:w14183.

16. Guinan P, Sobin LH, Algaba F, Badellino F, Kameyama S, MacLennan G, et al. TNM staging of renal cell carcinoma. Cancer 1997;80:992-3.

17. Wei SQ, Li CY, Li BG, Li JM, Wang H. UBE2C siRNA inhibits proliferation and invasion of human castration resistant prostate cancer PC3 cells. Tumor 2015;11:1200-7.

18. Wang HY, Zhang CP, Rorick A, Wu DY, Chiu M, ThomasAhner J, et al. CCI-779 inhibits cell-cycle G2/M progression and invasion of castration resistant prostate cancer via attenuation of UBE2C transcription and mRNA stability. Cancer Res 2011;71:4866-76.

19. Zhang HQ, Zhao G, Ke B, Ma G, Liu GL, Liang H, et al. Overexpression of UBE2C correlates with poor prognosis in gastric cancer patients. Eur Rev Med Pharmacol Sci 2018;22:1665-71.

20. Mo CH, Gao L, Zhu XF, Wei KL, Zeng JJ, Chen G, et al. The clinicopathological significance of UBE2C in breast cancer: a study based on immunohistochemistry, microarray and RNAsequencing data. Cancer Cell Int 2017; 17:83.

21. Hutson TE, Fiqlin RA. Renal cell cancer. Cancer J 2007; 13:282-6.

22. Yuan LS, Chen L, Qian KY, Qian GF, Wu CL, Wang XH, et al. Co-expression network analysis identified six hub genes in association with progression and prognosis in human clear cell renal cell carcinoma (ccRCC). Genom Data 2017;14:132-40.

23. Aze A, Maiorano D. Recent advances in understanding DNA replication: cell type-specific adaption of the DNA replication program. F1000Res 2018;7:F1000 Faculty Rev-1351.

24. Deneke VE, Puliafito A, Krueger D, Narla AV, De Simone A, Primo L, et al. Self-organized nuclear positioning synchronizes the cell cycle in drosophila embryos. Cell 2019;177:925-41.

25. Bjorklund M. Cell size homeostasis: metabolic control of growth and cell division. Biochim Biophys Acta Mol Cell Res 2019; 866:409-17.

26. Hanahan D, Weinberg RA. The hallmarks of cancer. Cell 2000;100:57-70.

27. Hanahan D, Weinberg RA. Hallmarks of cancer: the next generation. Cell 2011;144:646-74.

Received for publication: 9 November 2020. Accepted for publication: 10 February 2021

This work is licensed under a Creative Commons Attribution-NonCommercial 4.0 International License (CC BY-NC 4.0).

(C) Copyright: the Author(s), 2021

Licensee PAGEPress, Italy

European Journal of Histochemistry 2021; 65:3196

doi:10.4081/ejh.2021.3196 